\title{
Los deslizamientos de manifestación progresiva
}

\section{The slips of progressive manifestation}

\author{
Silvestre Elier Pacheco Moreno*, Alberto Lewis Concepción**, \\ Héctor Emilio Palza Arias-Barahona***
}

http://dx.doi.org/10.21503/CienciayDesarrollo.2006.v7.10

\section{RESUMEN}

El estudio se inició en el núcleo urbano del municipio Mariel, provincia de La Habana, Cuba. El área investigada ha estado sometida a la acción de las actividades humanas desde principios del siglo XIX, con predominio actual de un suelo urbanizado. Los objetivos fueron estudiar los factores susceptibles y la dinámica de los deslizamientos de tierra. La investigación fue planificada en tres etapas. En la primera, la bibliografía, los mapas y las fotografías aéreas fueron objeto de consultas y análisis; en la segunda fueron estudiados los elementos naturales susceptibles, en relación con la geomorfología, la geología y la hidrografía, así como con las acciones ejecutadas por el hombre; y la tercera etapa consistió en el control estadístico de las lluvias acaecidas y el monitoreo de los movimientos. Los resultados demostraron que las lluvias fueron el elemento disparador de la gradual sobresaturación de las arcillas entre dos formaciones geológicas terrígenas-carbonatadas. Los movimientos fueron lentos y prolongados, con intensidades de $9 \mathrm{~mm}$ /día y aperturas de grietas en terreno con magnitudes de $0,51 \mathrm{~m}$. Las infraestructuras en general fueron afectadas por agrietamientos, desplazamientos y derrumbes. La amenaza fue clasificada como de manifestación progresiva.

Palabras clave: deslizamientos, susceptibilidad, dinámica.

\section{ABSTRACT}

The study began in the urban nucleus of Mariel, County The Havana, Cuban. The investigated area was subjected to the action of the human activities from the beginning of the XIX century with current prevalence of an urbanized floor. The objectives were to study the susceptible factors and the dynamic of the slips of earth. This investigation was planned in three stages: the bibliographies, the maps and the air pictures were objects of consultations and analysis in the first; in the second the susceptible natural elements conditioned with relationship to the geomorphology were studied, geology and hydrograph as well as the actions executed by the man; the third stage consisted of the statistical control of the rains and the monitors of the movements. The results demonstrated that the rains were the element trigger that they rebounded gradual modifying the clays between the two geological formations of form terrifiescarbonated. The movements were slow and lingering with intensities of 9 millimeters/day and openings of cracks in land with magnitudes of 0.51 meters.. The infrastructures in general were affected for cracklings, displacements and knock down. The threat was classified of progressive manifestation.

Keywords: slips, susceptibility, dynamic.

\footnotetext{
* Instituto de Geofísica y Astronomía, Departamento de Estudios Geoambientales y Riesgos. Ciudad de La Habana, Cuba.

"Instituto de Geofísica y Astronomía, Departamento de Estudios Geoambientales y Riesgos. Ciudad de La Habana, Cuba.

"** Universidad Nacional de San Agustín de Arequipa.
} 


\section{INTRODUCCIÓN}

La investigación se realiza en el núcleo urbano de Mariel, provincia de La Habana perteneciente a la región occidental de Cuba. El lugar posee una historia con antecedentes de deslizamientos de tierra que provocaron en diferentes períodos la evacuación de miles de personas y numerosas pérdidas materiales. Estos eventos naturales extremos propios de zonas montañosas se manifiestan de forma súbita, con excepción del caso presentado en las llanuras altas costeras ( 90 $\mathrm{m}$ ), en el que se produjeron movimientos muy lentos. (Pacheco y Lewis, 1998; Pacheco, 2003, 2004, 2005.)

La importancia del estudio radica en conocer las manifestaciones atípicas de los movimientos de masas de tierra en esta localidad, el aumento de la susceptibilidad, la dinámica progresiva y su potencialidad destructiva.

Los resultados demostraron que los deslizamientos son rotacionales-translacionales, con dirección hacia la bahía de Mariel, y que el aumento de la susceptibilidad del área se dio por procesos hidrogeológicos-geomorfológicos favorecidos por la acción negativa del hombre. Por otra parte, las lluvias acumuladas fueron el elemento disparador de la alteración gradual de las arcillas motmorillonitas yacentes entre las formaciones geológicas predominantes.

La frecuencia de los eventos estuvo relacionada con los períodos climáticos muy lluviosos, y la intensidad de los movimientos llegó a ser de hasta $9 \mathrm{~mm} /$ día, provocando grietas primarias y secundarias en el terreno que alcanzaron magnitudes de $0,51 \mathrm{~m}$.

Las edificaciones y los sistemas de redes aéreas del servicio eléctrico y telefónico fueron dañados por agrietamientos, corrimientos, derrumbes e inclinaciones. Las redes del servicio de agua potable a la población fueron partidas por los movimientos de masas. Los deslizamientos fueron clasificados de manifestación progresiva.

\section{MATERIAL Y MÉTODOS}

La investigación en su primera fase se dirigió hacia el trabajo de gabinete. Se consultó la bibliografía y se participó en talleres y eventos con grupos de expertos en la materia a nivel nacional e internacional (Geominería, 1998; CISMID, 2003; Geoinfo, 2004; Geominería, 2005; Convención del Medio Ambiente, 2005), privilegiando el intercambio de ideas.

Los mapas, fotografías aéreas y parcelarios a escalas 1:10 000, 1:5 000, 1:2 000 (DMPF, 2004; ICGC, 1982) permitieron observar, analizar y comparar las variaciones en la topografía, la red hidrográfica, la geología, el uso del suelo urbano y las acciones ejecutadas por el hombre. También se consultaron calas realizadas (ENIA, 1994; Pérez et al, 1988) para comprobar la estratigrafía y la litología de las diferentes formaciones geológicas. Se analizaron la estadística de lluvias acaecidas por más de dos décadas (INRH, 2005) y su vínculo con eventos meteorológicos extremos. Fue tomado el Parámetro Indicador de Alerta Temprana (Pacheco, 1994) a partir de 1 150,00 mm de lluvias acumuladas y se estableció el monitoreo estadístico-instrumental aplicando la técnica de estacas orientadas para grietas en terreno y la de reglas graduadas metálicas para grietas en estructuras de hormigón armado.

\section{RESULTADOS}

Los deslizamientos encontrados en el Municipio de Mariel fueron los primeros identificados en llanuras altas costeras (menos de $120 \mathrm{~m}$ ) en la región occidental de Cuba, y están valorados como atípicos por su dinámica gradual, lenta y prolongada. 


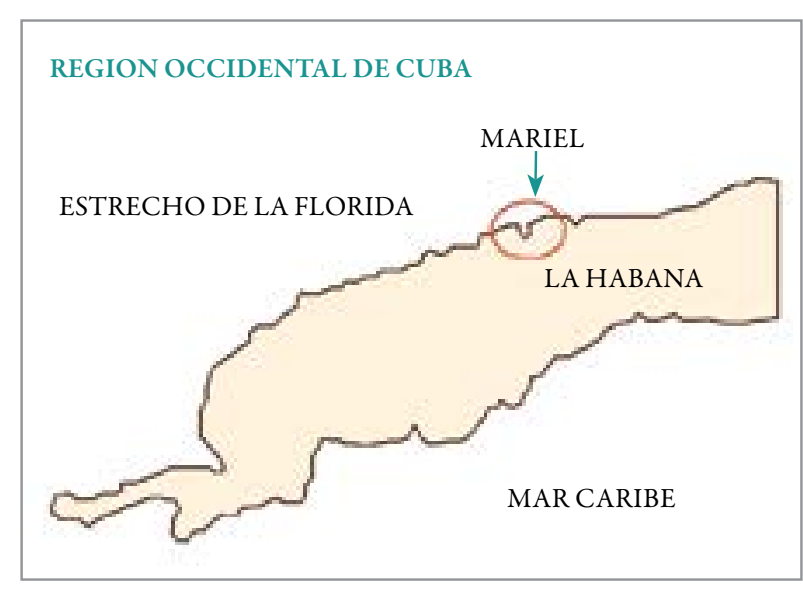

Figura 1. Ubicación geográfica del Municipio de Mariel.

Los factores naturales que propiciaron la susceptibilidad del área fueron la presencia de formaciones geológicas terrígeno-carbonatadas (Universidad y Vía Blanca), una falla en paralelo a las formas convexas del relieve y la acción de las aguas subterráneas de estratos fisurales. Otros factores considerados de gran peso fueron los originados por el hombre, como el cierre del antiguo acueducto, la ampliación del puerto, los cortes de taludes y la deforestación.

Los períodos muy lluviosos fueron considerados como la amenaza principal, siempre que las estadísticas sobrepasen la lámina de 1150,00 milímetros acaecidos.

Las argilitas (arcillas gris-verde oscuro) fueron el elemento litológico modificado del medio que provocó una dinámica lenta y progresiva,

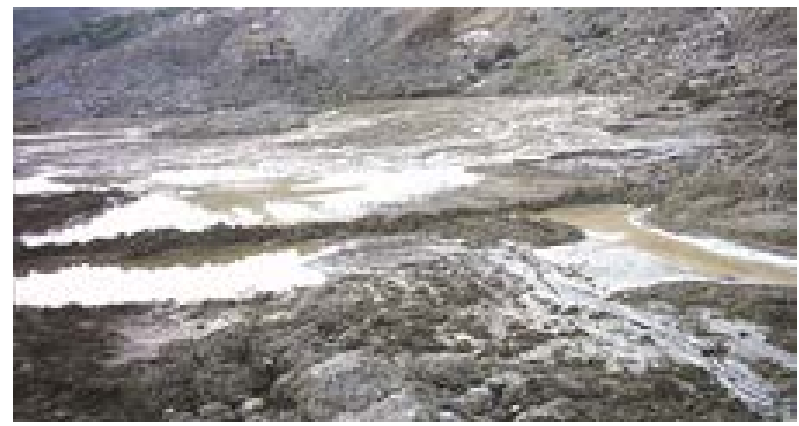

Figura 2. Argilitas afloradas por la elevación del nivel freático. con intensidades máximas de $9 \mathrm{~mm} /$ día para magnitudes en grietas de $0,51 \mathrm{~m}$. La figura 2 muestra las argilitas afloradas y sobresaturadas por la elevación del nivel freático, las mismas que provocaron encharcamientos en una cantera de extracción de materiales para la construcción, a 1 $\mathrm{km}$ del lugar de los deslizamientos.

Las edificaciones y sistemas de redes aéreas sufrieron afectaciones lentas por agrietamientos, corrimientos, inclinaciones y derrumbes. Las redes telefónicas soterradas fueron cubiertas por las aguas subterráneas de los manantiales.

\section{DISCUSIÓN}

La confirmación de la presencia de los cinco deslizamientos de tierra (Pacheco y Lewis, 1998) en la periferia del núcleo urbano de Mariel hizo evidente que la magnitud del peligro era mayor al en estudios anteriores identificado (Albear, et al, 1968 y ENIA, 1984). Estos eventos naturales extremos causados por intensas lluvias son propios de zonas montañosas con pendientes abruptas, y producen avalanchas que son considerados como peligros de manifestación súbita. Sin embargo, el caso de Mariel se manifestó de forma atípica en un relieve de llanuras altas costeras $(90,00 \mathrm{~m})$ con pendientes suaves. No se produjeron avalanchas, y sus movimientos dinámicos fueron graduales, lentos y prolongados. La figura 3 muestra la dirección de los deslizamientos hacia el núcleo urbano y la bahía.

El estudio de los factores susceptibles permitió conocer los elementos naturales condicionados y la acción negativa del hombre. Entre los factores naturales encontrados hay dos formaciones geológicas (Universidad y Vía Blanca). La formación Universidad, del eoceno inferior a medio, está constituida de forma general por margas masivas blancas y amarillentas que transicionan hasta arcillas calcáreas. Por su parte, la formación Vía Blanca, del cretácico superior, es predominante 


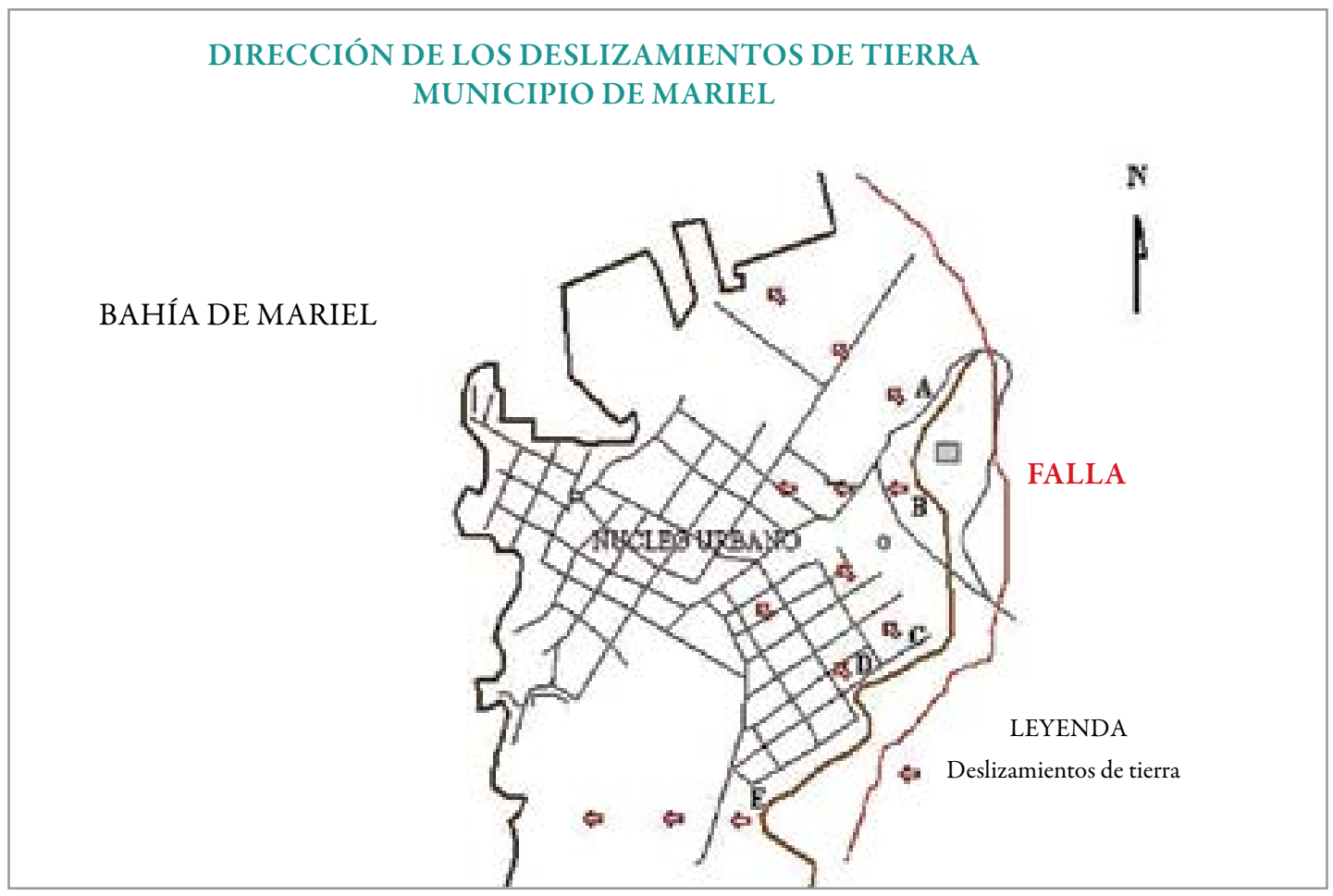

Figura 3. Dirección de los deslizamientos de tierra.

en argilitas (arcillas motmorillonitas). Ambas formaciones son terrígeno-carbonatadas y yacen una sobre otra en caída angular, con buzamientos de 30 a 35 grados hacia la bahía.

En el estudio tectónico encontramos varias fallas, pero una fue localizada encima de la elevación, con una orientación NW-SW. Esta dista aproximadamente de 50 a $70 \mathrm{~m}$ de las formas convexas que describe el relieve, coincidiendo con los deslizamientos de tierra (Pacheco y Lewis, 1998).

La elevación es atravesada por las aguas subterráneas que fluyen de forma libre a través de la falla y los estratos de las formaciones geológicas predominantes. Esto, favorecido por los períodos lluviosos prolongados, ha sido considerado como la amenaza principal al manto freático y como causa de alteración de la litología. Los deslizamientos fueron causados por la sobresaturación de las argilitas (arcillas motmorillonitas) de color gris-verde oscuro, de forma angular, yacentes entre las formaciones geológicas.
Tras el estudio de los factores naturales, se analizó e investigó la actividad humana que influyó de forma negativa. La ampliación del puerto (año 1956) y el cierre de los manantiales del acueducto municipal (década 1930-40) provocaron la obstrucción de la salida de las aguas subterráneas a la bahía. Otros factores negativos fueron el crecimiento urbano y la deforestación progresiva desde principios del siglo $\mathrm{XX}$, que afectaron especialmente a los taludes.

Los resultados obtenidos del estudio de susceptibilidad demostraron la presencia de un proceso hidrogeológico-geomorfológico condicionado como causa endógeno-exógena, sumada a la acción antrópica centenaria.

En la tabla 1 se exponen los resultados de los monitoreos basados en el control de lluvias acaecidas y en la apertura de grietas, quedando demostrada la validación de 1 150,00 milímetros como Parámetro Indicador de Alerta Temprana (PIAT). 
Tabla 1 (septiembre de 2005). Monitoreo del deslizamiento B (metros)

\begin{tabular}{l|c|c|}
\multicolumn{1}{c|}{ Fecha } & Lluvias acaecidas & $\begin{array}{c}\text { Magnitud de la } \\
\text { grieta }\end{array}$ \\
\hline 20 de septiembre & 1173,6 & 0,05 \\
\hline 21 & 1314,6 & 0,12 \\
\hline 22 & 1334,6 & 0,24 \\
\hline 8 de octubre & 1384,7 & 0,35 \\
\hline 18 & 1397,1 & 0,42 \\
\hline 21 & 1425,1 & 0,48 \\
\hline 22 & 1425,1 & 0,51 \\
\hline
\end{tabular}

En el gráfico de la figura 4, se muestra la curva ascendente de la dinámica de los movimientos en función del aumento de la lámina de lluvia. Las magnitudes de las grietas en el terreno como causa de la expansión de la masa fueron de 0,05 hasta $0,51 \mathrm{~m}$, y la intensidad máxima registrada en el último período (septiembre-octubre de 2005)

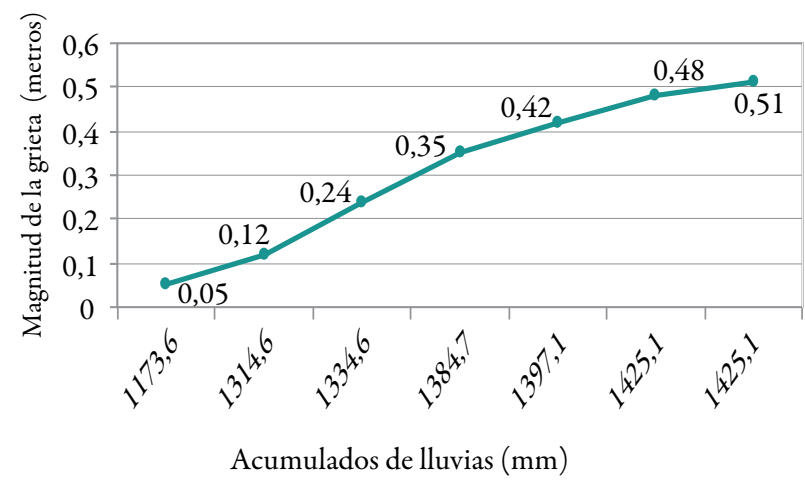

Figura 4. Magnitudes de las grietas en función de las láminas de lluvias.

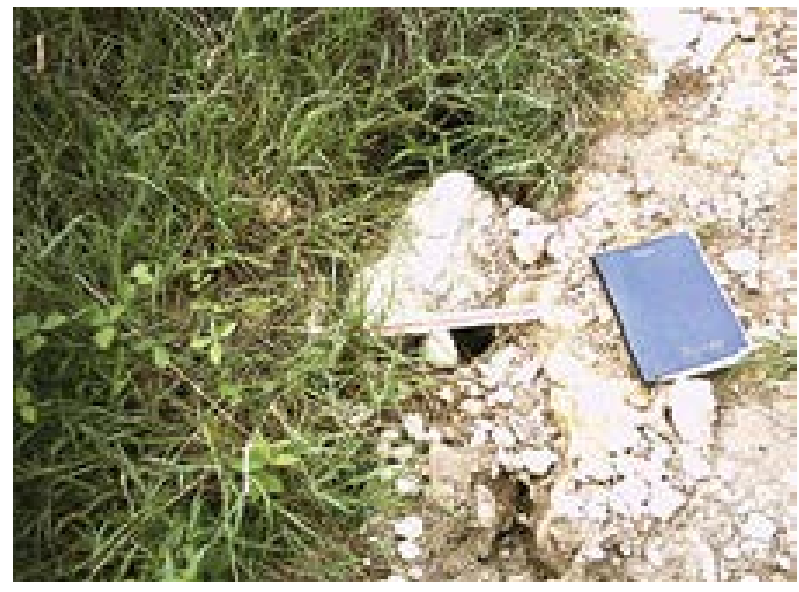

Figura 5. Grieta de 0,33 m. estuvo en $9 \mathrm{~mm} /$ día, siendo valorada la amenaza de manifestación progresiva.

El patrimonio estructural construido, como son las residencias, locales administrativos, almacenes y otros ubicados en el radio de acción de los movimientos, sufrió gradualmente agrietamientos, corrimientos y derrumbes. Las redes eléctricas, telefónicas y sanitarias también sufrieron severos impactos.

\section{CONCLUSIONES}

1. Los deslizamientos encontrados son rotacionales-translacionales y se desarrollaron de forma atípica en llanuras altas costeras (90 m) de la región occidental de Cuba.

2. La susceptibilidad del terreno fue condicionada integralmente por factores naturales y antrópicos, siendo las argilitas (arcillas motmorillonitas) el elemento modificado por los períodos lluviosos.

3. Los monitoreos demostraron que los movimientos fueron lentos, prolongados y graduales, con intensidades máximas de $9 \mathrm{~mm} /$ día. No fueron registradas avalanchas, y la masa de tierra avanzó en función de la lámina de lluvia acaecida.

4. La amenaza fue clasificada como Deslizamientos de Manifestación Progresiva.

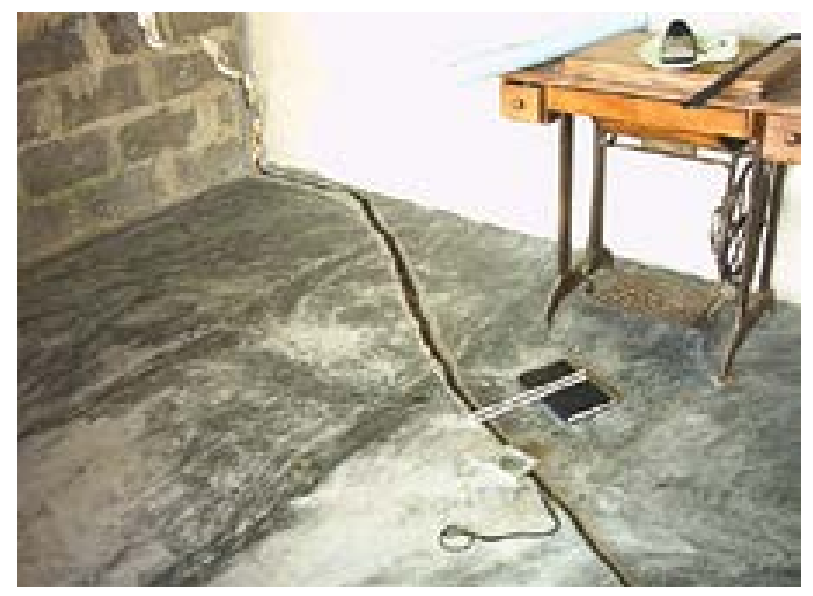

Figura 6. Muestra de cómo una grieta afectó a una residencia. 


\section{REFERENCIAS BIBLIOGRÁFICAS}

1. Díaz J. L, et al. Los principios básicos de la clasificación morfoestructural del relieve cubano y su aplicación en la región centro oriental. Instituto de Geografía, Academia de Ciencias, La Habana, Cuba, 1986, 43 pp.

2. Dirección Municipal de Planificación Física de Mariel. Unidad Básica de Información Territorial. Parcelarios 1:10000, 1:50000, 1:2000. La Habana, Cuba, 2004, 32 pp.

3. Empresa Nacional de Investigaciones Aplicadas. Establecimiento de Investigaciones Marítimas. Informe Ingeniero Geológico. Astilleros Mariel. La Habana, Cuba, 1994, 10 pp.

4. Fondo Geológico. Mapa de formaciones geológicas de la provincia La Habana. Oficina Nacional de Recursos Minerales, Ciudad de La Habana, Cuba, 2003, 1pp.

5. Hernández Santana, J.R, et al. "Estilos geotectónicos bidimensionales y tridimensionales interbloques: una nueva categoría neotectónica para la determinación de morfoestructuras montañosas”. Investigaciones Geográficas. Boletín del Instituto de Geografía. No 28, México, 1999, 10 pp.

6. Instituto Cubano de Geodesia y Cartografía. Atlas de Cuba. XX Aniversario del Triunfo de la Revolución. La Habana, Cuba, 1978, pp. $24-25$.

7. Recursos Hidráulicos. Control estadístico de lluvias. Estación 355, 394 y 400. Correo Mariel, La Habana, Cuba, 2005, 12 pp.
8. Pérez, E. Informe (argilitas). Ministerio de la Industria de los Materiales de la Construcción. Empresa Geólogo-Minera, Ciudad de La Habana, Cuba, 1988, 176 pp.

9. Pacheco, S. E. La meteorología en la prevención de los desastres. Volumen 1. VIII Congreso Brasilero de Meteorología y II Congreso Latinoamericano e Ibérico de Meteorología. Belo Horizonte, Brasil, 1994, pp. $337-342$.

10. Pacheco, S. E., A. Lewis. "Factores que originan los deslizamientos de tierra. Afectaciones en el municipio de Mariel". Memorias de Geología y Minería 98. Tomo I. Centro Nacional de Información Geológica, La Habana, Cuba, 1998, pp. 228 - 531.

11. Pacheco S.E. Los peligros naturales como potenciales destructores. Afectaciones urbanisticas. Seguimiento de caso. Centro Peruano Japonés de Investigaciones Sísmicas y Mitigación de Desastres (CISMID), Lima, Perú, 2003, 18 pp.

12. Pacheco S E. Análisis de la susceptibilidady dinámica de zonas deslizantes en la localidad de Mariel. VII Taller Internacional Informática y Geociencias, Cuba, 2004, 16pp.

13. Palza H, E. Zonas de alto riesgo de la Cuarta Dirección Regional de Defensa Civil (Cusco, Apurimac y Madre de Dios). Centro Peruano Japonés de Investigaciones Sísmicas y Mitigación de Desastres (CISMID), Lima, Perú, 2003. 